\title{
DOUBLE-PERIOD OBSERVATIONS OF THE EMISSION FROM PSR 1133+16 AT LOW FREQUENCIES
}

\author{
YU. M. BRUCK \\ Institute of Radio Astronomy
}

\section{Introduction}

Observations with double period integration $\left(2 P_{\text {obs }}\right)$ were used for the first time in 1972 to provide reliable detection of pulsars at low frequencies (Ustimenko 1983), where $P_{\text {obs }}$ is the observed period. In 1973 observations of PSR $1919+21$ were conducted with the period $4 P_{\text {obs }}$, during which interpulse emission (IPE) of that pulsar was found (Bruck and Ustimenko 1977b), later identified with observations at $38 \mathrm{MHz}$ (Bash, Bozyan, and Torrance 1970, Bruck and Ustimenko 1977a). Later, because of the lower resolution, this method was not used, and its obvious advantages were forgotten. We returned to observations with $2 P_{\text {obs }}$ integration period in 19837 to detect new pulsars, which proved necessary to overcome a number of difficulties and contradictions in identification of IPE. The detection reliability requires a high signal-to-noise ratio and a repetition of the result. For a stationary signal located in the limited region of pulsar longitude, this is achieved by increasing the receiving bandwidth and the observation time. For the sporadic IPE such averaging leads to a considerable loss of information and requires complex methods to be used for identifying signals. The method discussed is free from all these drawbacks; we shall present some results of its use for investigating PSR $1133+16$ in the $25 \mathrm{MHz}$ range. The observation method and the equipment parameters differed from those described in the preceding report (Bruck and Ulyanov 1992) only in the integration cycle $\left(2 P_{\text {obs }}\right)$. Both methods of data processing were used.

\section{Linear processing}

After storage of a number of pulses, the signal $F(t)$ $\left(0 \leq t \leq 2 P_{\text {obs }}\right)$ was divided into two parts: $F_{1}\left(t_{1}\right)$ $\left(0 \leq t_{1} \leq P_{\text {obs }}\right)$ and $F_{2}\left(t_{2}\right)\left(P_{\text {obs }} \leq t_{2} \leq 2 P_{\text {obs }}\right)$. Signals for the sum, $S(t)=0.7\left(F_{1}\left(t_{1}\right)+F_{2}\left(t_{2}\right)\right)$ and the difference, $N(t)=0.7\left(F_{1}\left(t_{1}\right)-F_{2}\left(t_{2}\right)\right)$ were calculated. Then $F(t)$ and $S(t)$ were normalized using $G_{\mathrm{n}}$, the root mean square value of $N(t)$ or $G_{f}$, the root mean square noise value determined before the storage of signals. As $N(t)$ includes the non-correlated signal fluctuations and occasional interference, $G_{\mathrm{n}}>G_{\mathrm{f}}$ (usually $G_{\mathrm{n}} \geq 1.5 G_{\mathrm{f}}$ ), this normalization excludes the probability of false detection. Then the peak-to-peak amplitude, $A$, and cross-correlation coefficient, $R$, of the signals $F_{1}\left(t_{1}\right)$ and $F_{2}\left(t_{2}\right)$ were calculated. The detection was considered reliable if $A / G_{\mathrm{n}} \geq 6$ or $R \geq 0.5$. The processing was conducted both for this total signal and for the signal more than $\pm 45^{\circ}$ from the main pulse maximum, taken as a zero. The given interval is 2 times wider than its true width determined with the average profile. Figure 1 shows an example of the

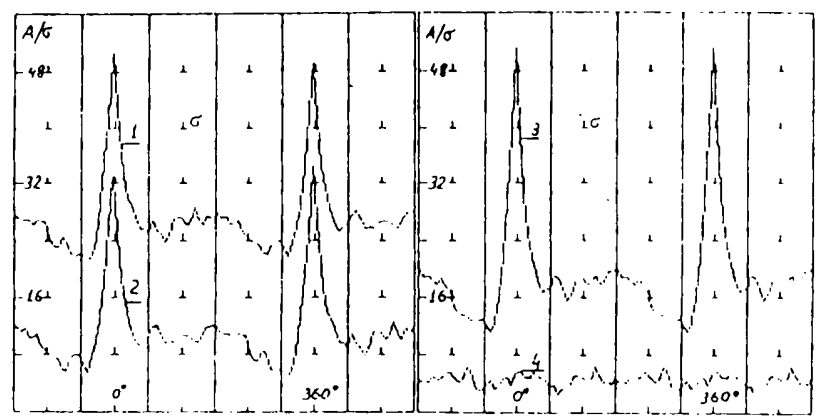

Figure $125-\mathrm{MHz}$ IPE for PSR $1133+16$ in the average of $1800 * 2 P_{\text {obs }}$. Curve 1 is $F(t), 2$ is $F(t+T), 3$ is $S(t)$, 4 is $N(t), A=45(9)$ and $\mathrm{R}=0.97(0.67)$. The IPE values are shown in the brackets. 12 channels over $112-\mathrm{kHz}$ bandwidth, 49 points per period.

detection of IPE after the accumulation of $1800 * 2$ periods over a $100-\mathrm{kHz}$ band. One can see here clear maxima near the main pulse, the radiation minimum and the extended component. Figure 2 shows

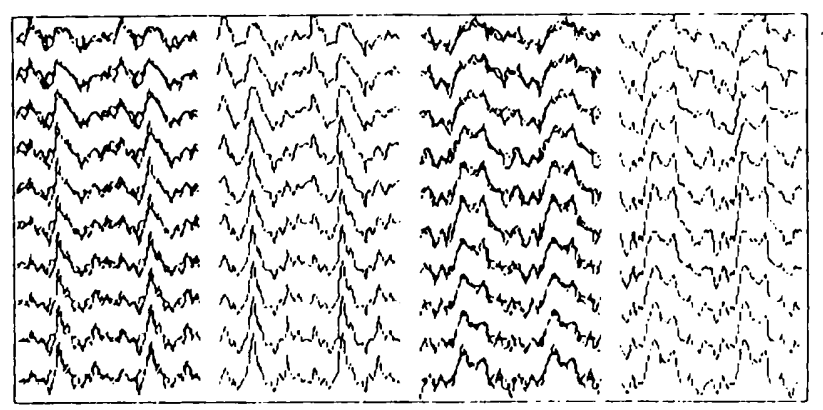

Figure 2 Two examples of detecting the IPE for $100 *$ $2 P_{\text {obs }}$ in 12 receiving channels.

two examples of detecting the IPE for $100 * 2$ periods in 12 receiving channels. For a convenient comparison of the signals $F(t)$ and $F(t+T)$, they are superposed on the $y$-coordinate. Coincidence of most of the IPE features and their dispersion measure 
can be seen, the main pulse range being up to $140^{\circ}$ and the high correlation of the IPE: $A=16(10)$, $R=0.7(0.48)$.

\section{Correlation analysis}

Correlation analysis applied to the double period observations yields a unique opportunity to obtain an objective, statistically significant answer to most of the questions arising when investigating the IPE. Using the available data, one may calculate quite a lot of correlation matrices and correlation functions. We consider here only the following three cyclic auto-correlation functions:

$$
\begin{aligned}
& A_{\mathrm{f}}(h)=\langle F(t) * F(t+T)\rangle,(0 \leq h \leq T), \\
& A_{\mathrm{s}}(h)=\langle S(t) * S(t+h)\rangle, \\
& A_{\mathrm{n}}(h)=\langle N(t) * N(t+h)\rangle
\end{aligned}
$$

and the cyclic cross-correlation functions:

$$
A_{c}(h)=\left\langle F_{1}\left(t_{1}\right) * F_{2}\left(t_{2}+h\right)\right\rangle \text {. }
$$

Figure $3(1,2,3,4)$ show the given profiles, obtained

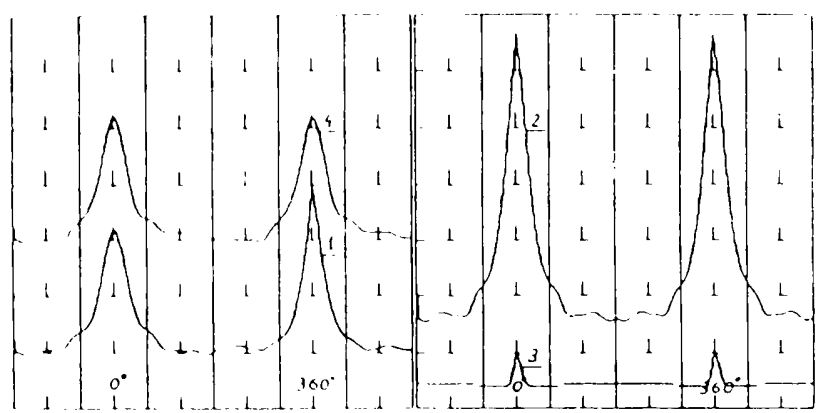

Figure $\mathbf{3}$ Profiles obtained by averaging 216 observations, independent of time and frequency.

by averaging 216 initial observations independent of time and frequency. In each of them, $100 * 2$ periods have been summed. We shall give the main results following from the analysis of these dependencies.

1. As $A_{\mathrm{f}}(t)=A_{\mathrm{c}}(0)=\max$, one may assume that the signals of $F_{1}\left(t_{1}\right)$ and $F_{2}\left(t_{2}\right)$ are identical with accuracy up to the noise.

2. The secondary maxima $A_{\mathrm{f}}(h)$ and the main maxima $A_{s}(h), A_{c}(h)$ seem to consist of two components of different widths. The charac- teristic sizes of these IPE regions are of $45^{\circ}$ and $140^{\circ}$. For the present it is not clear whether they are related, whether they exist together or separately and what coordinates they have.

3. The $A_{\mathrm{c}}(0)$ values determined for the presence and absence of the the main pulse give separately its energy and the energy of all the correlated components of the IPE. Their ratio depends on a number of stored pulses, which, on the average is $>100 \%$ of the main pulse energy.

4. The spectrum $A_{\mathrm{n}}(h)$ coincides with the frequency characteristics of the equipment and the processing system which proves the high identity of the signals of $F_{1}\left(t_{1}\right)$ and $F_{2}\left(t_{2}\right)$. $A_{n}(0)$ gives the values of the additive noise and the signal fluctuations. Absence of the $A_{\text {n }}(h)$ fluctuations confirms sufficiently the averaging procedure and a high degree of reliability of the other correlation functions.

Finally let us consider a question on the IPE observation probability. For this we turn to figure 4 which presents the distribution histograms of the

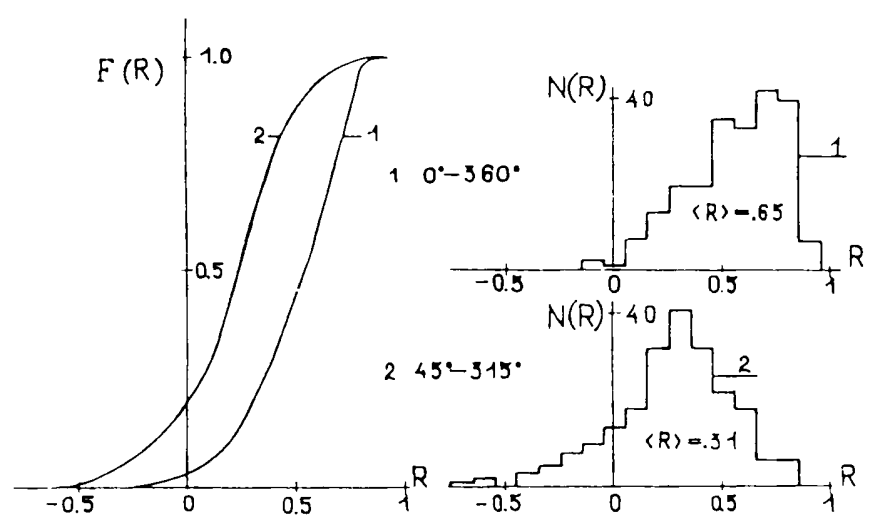

Figure 4 Distribution histograms of the cross-correlation coefficients of $F_{1}\left(t_{1}\right) * F_{2}\left(t_{2}\right)$, a. Main pulse; $b$. Without the main pulse.

cross-correlation coefficients of $F_{1}\left(t_{1}\right) * F_{2}\left(t_{2}\right)$ and the integration laws corresponding to them for the signals with the main pulse $(a)$ and without it $(b)$. It is seen that the mean values are displaced into the region of the positive correlation. The distribution law is close to Fisher's distribution for the correlated random process. 